\title{
Simulations of Weak Gravitational Lensing
}

\author{
Martin White ${ }^{\mathrm{a}, \mathrm{b}, 1}$, Chris Vale ${ }^{\mathrm{b}, 2}$

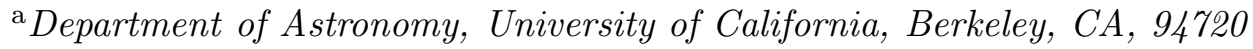 \\ ${ }^{\mathrm{b}}$ Department of Physics, University of California, Berkeley, CA, 94720
}

\begin{abstract}
We describe the simulation data produced by a pilot programme to compute mock weak gravitational lensing maps for a range of currently popular cosmological models by ray tracing through high-resolution N-body simulations. The programme required only a modest investment in computer time to produce maps accurate to arcminute scales covering hundreds of square degrees of sky for 4 cosmological models.
\end{abstract}

Key words: Cosmology, Lensing, Large-Scale structures

PACS: 98.65.Dx, 98.80.Es, 98.70.Vc

\section{Introduction}

Weak gravitational lensing by large-scale structure has become an indispensable tool for modern cosmology, used already to set constraints on the mass density $\left(\Omega_{\text {mat }}\right.$, in units of the critical density) and the fluctuation amplitude $\left(\sigma_{8}\right)$ (see e.g. van Waerbeke \& Mellier, 2003; Hoekstra, Yee, \& Gladders, 2002, for the current status) and touted for its potential to constrain cluster scaling relations (Huterer \& White, 2002) and dark energy (Benabed \& Bernardeau, 2001; Huterer, 2002; Hu, 2002; Heavens, 2003; Abazajian \& Dodelson, 2003; Refregier, 2003; Jain \& Taylor, 2003; Bernstein \& Jain, 2004; Takada \& Jain, 2004; Takada \& White, 2004). Like the anisotropies in the cosmic microwave background (CMB), the theory of weak gravitational lensing is well understood (Mellier, 1999; Bartelmann \& Schneider, 2001). Unlike the CMB however the calculation involves modeling the non-linear evolution of the mass in the universe. This makes the predictions of weak lensing very rich, but also means that an accurate treatment requires numerical simulations.

1 E-mail: mwhite@astro.berkeley.edu

2 E-mail: cvale@astro.berkeley.edu

Preprint submitted to Elsevier Science 29 October 2018 
In this paper we give some details of a pilot program designed to provide weak lensing ray-tracing simulations of a small grid of cosmological models in the currently favored region of parameter space. These models can be used by observers wishing to test their algorithms or fit to their data and by theorists wishing to test or calibrate fast and flexible, but approximate, methods of calculation. The initial grid is small, but the process of its creation is almost entirely automatic allowing it to be expanded simply as the need arises.

This paper focuses on the creation of the model grid. We describe the choice of cosmological parameters in $\S 2.1$, the N-body simulations in $\S 2.2$ and the ray tracing and numerical issues in $\S 2.3$ and $\S 2.4$. We give some representative results in $\S 3$ before concluding in $\S 4$.

\section{Cosmological models}

\subsection{Choosing parameters}

We choose a small number of cosmological models which provide good fits to the current CMB and large-scale structure data. For simplicity all of the models in the pilot program are variants of the cold dark matter model with a dark energy component, and their parameters are shown in Table 1. The parameter variations around a base model are designed to keep the CMB fluctuations almost invariant, in anticipation of increasingly precise data from WMAP and Planck, and the entire process is automated using Perl scripts and $\mathrm{C}$ code.

We begin with a relatively small number of models to demonstrate the cost and feasibility of making such grids. As we gain experience in using these data products and understand the drivers better we can extend the model grid and/or increase the fidelity of the simulations. Are our priorities to have larger maps? higher resolution? more redshift range? more 'sky'? etc.

We first pick the physical matter density $\omega_{\text {mat }} \equiv \Omega_{\text {mat }} h^{2}$, the (comoving) distance to last scattering, $d_{\mathrm{ls}}$, and a (constant) equation of state of the dark energy, $w$. We approximate the redshift of last scattering as $z=1080$, ignoring the slight matter and baryon density dependence. This then allows us to solve for the Hubble constant, $h$, and thus $\Omega_{\text {mat }}$ and $\Omega_{\text {de }}$ assuming spatial flatness. Because they are reasonably well known, compared to some other parameters, we fix $\omega_{\text {mat }}=0.145$ and $d_{\mathrm{ls}}=13.7 \mathrm{Gpc}$ for all of the models in our grid. These numbers are close to the best fit for a recent analysis of WMAP and SDSS data (Tegmark, 2003). 


\begin{tabular}{cccccccc} 
Model & $\Omega_{\text {mat }}$ & $w$ & $h$ & $n$ & $\sigma_{8}$ & $\tau$ & $\chi^{2}$ \\
\hline $1 \& 2$ & 0.296 & -1.0 & 0.70 & 1.00 & 0.93 & 0.15 & 977 \\
$3 \& 4$ & 0.357 & -0.8 & 0.64 & 1.00 & 0.88 & 0.15 & 975 \\
$5 \& 6$ & 0.296 & -1.0 & 0.70 & 0.95 & 0.85 & 0.10 & 979 \\
$7 \& 8$ & 0.357 & -0.8 & 0.64 & 0.95 & 0.81 & 0.10 & 976
\end{tabular}

Table 1

Parameters for the models run. For each cosmological model two independent sets of initial conditions are generated. All models are spatially flat, so $\Omega_{\mathrm{de}}=1-\Omega_{\mathrm{mat}}$. For all models the matter density is $\omega_{\text {mat }}=0.145$ and the baryon density $\omega_{b} \equiv \Omega_{b} h^{2}$ was fixed at 0.023 . Our results are very insensitive to this latter choice. All models have power-law spectra (no running) with index $n$ and the dark energy has a constant equation of state $w$. The normalization, $\sigma_{8}$, is from a fit to the WMAP TT power spectrum data and the $\chi^{2}$ is for this fit with 893 degrees of freedom.

The next step is to specify the other model parameters, for example the optical depth to Thomson scattering, $\tau$, the spectral index, $n$ and the baryon density $\omega_{b}$. (The optical depth enters primarily through its effect on the normalization of the power spectrum when we fit to WMAP.) We currently hold the number of light neutrinos fixed, and include no massive neutrinos. We deal with pure power-law spectra with no running spectral index. Our primary variations are in the optical depth, the spectral index and the equation of state of the dark energy (see Table 1). The first two affect the amplitude and shape of the density or potential fluctuations at late times while $w$ is a parameter of great interest to the cosmology community. We use a fixed equation of state in this initial survey, though it is easy to include any known functional form in the future.

For a specified model we compute the CMB anisotropy spectrum using v4.5 of CMBFAST (Seljak \& Zaldarriaga, 1996). The likelihood software provided by the WMAP team (Verde et al., 2003; Hinshaw et al., 2003; Kogut et al., 2003) and the known anisotropy spectrum are then used to find the best fitting normalization of the power spectrum, $\sigma_{8}$, which is then converted into internal code units for the N-body simulations. In this way a file of model parameters, shown in Table 1, is built up. The lensing convergence angular power spectra for our 4 models, assuming the Born and Limber approximations, are shown in Fig. 1. Note that the spectra for each pair of models have similar shapes, differing primarily in amplitude. In each case the model with the most distant sources has the highest amplitude, even though it has a slightly lower matter power spectrum amplitude, $\sigma_{8}$. There will also be differences in the higher order moments and the source redshift dependence of the power spectra. 


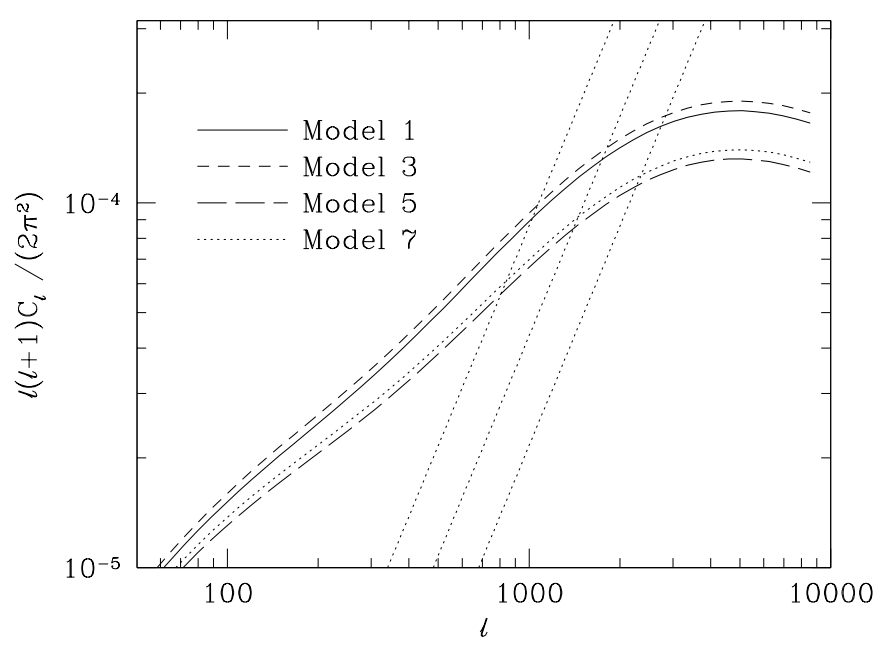

Fig. 1. The angular power spectra predicted for our 4 cosmological models for sources at $z_{s} \equiv 1$. We assumed the Born and Limber approximations, and used an ansatz for the non-linear mass power spectrum to make these predictions. The dotted lines rising steeply to top right are the shot-noise power spectra for $\gamma_{\mathrm{rms}}=0.4$ and $\bar{n}_{\text {gal }}=25,50$ and $100 \operatorname{arcmin}^{-2}$.

\subsection{N-body simulations}

Another Perl program reads this cosmological parameter file and generates a directory structure containing the requisite auxiliary files for the N-body simulation, plus scripts for generating the initial conditions and running the N-body code on the IBM-SP Seaborg at NERSC.

All simulations require choices to be made about box size, resolution and sampling. To minimize numerical artifacts we want to run the largest box with the highest dynamic range consistent with the computational resources. Since this is a pilot program we have chosen simulations which use $256^{3}$ particles in a $200 h^{-1} \mathrm{Mpc}$ box. As we shall discuss in $\S 2.4$, this is expected to be sufficient for our purposes now, but will need to be improved upon in the future. As further resources become available we can increase the volume simulated and/or decrease the force softening.

The initial conditions are generated by displacing equal mass particles from a "fuzzy" Cartesian grid at $z=50$ using the Zel'dovich approximation. The initial fluctuations are Gaussian, however we choose from a larger number of initial conditions those whose low few $k$-modes are close to the mean. This avoids running any simulations which are significant outliers and may skew statistics for a small number of runs. For each cosmological model we choose two independent initial conditions. Ideally we would run more realizations of each model to increase the volume simulated, but with two runs we will be 
able to simulate several hundred square degrees which is enough for now.

The TreePM code, described in White (2002), is used to evolve the particles to $z=0$ with the phase space information output at selected times after $z=3$. The outputs are spaced equally in conformal time, with a spacing equal to the time taken for light to travel $50 h^{-1} \mathrm{Mpc}$ (comoving). This spacing is small enough to obtain good lensing maps - a halo with "typical" velocity $300 \mathrm{~km} / \mathrm{s}$ will move only $50 h^{-1} \mathrm{kpc}$ between outputs, and its departure from straight line motion will be small. Each simulation gives between 80 and 90 phase space dumps, depending on the cosmology, each of $\sim 500 \mathrm{MB}$. The gravitational force softening is of a spline form, with a "Plummer equivalent" softening length of about $30 \mathrm{~h}^{-1} \mathrm{kpc}$, again small enough for our purposes.

Since the box size is only $200 h^{-1} \mathrm{Mpc}$, leading to a relatively coarse sampling in $k$, we generate the initial conditions from a fit to the transfer function which has the baryon oscillations "smoothed out". For now we use the fit published by Eisenstein \& Hu (1999). This fit was made to an older version of CMBFAST, and since that time improvements in the code and the physics (Seljak et al., 2003) have changed the predictions for $T(k)$ slightly. The differences are small, and for the purposes of testing and calibrating algorithms it is enough to know the input $T(k)$ precisely, but in the future we will improve this aspect.

\subsection{Making lens maps}

We make two sets of maps. The first, which we shall call the Born series, assumes that the convergence field, $\kappa$, is simply the integral along the line of sight of the density field weighted by a simple kernel

$$
\kappa \simeq \frac{3}{2} H_{0}^{2} \Omega_{\mathrm{mat}} \int d \chi g(\chi) \frac{\delta}{a}
$$

where $\delta$ is the overdensity, $a$ is the scale-factor, $\chi$ is the comoving distance and $g(\chi)$ is the lensing weight

$$
g(\chi) \equiv \int_{\chi}^{\infty} d \chi_{s} p\left(\chi_{s}\right) \frac{\chi\left(\chi_{s}-\chi\right)}{\chi_{s}}
$$

for sources with distribution $p\left(\chi_{s}\right)$ normalized to $\int d p=1$. This set of maps has the advantage of not requiring a Fourier transform in its construction, providing higher resolution for a given pixelization. Its principal disadvantage is the approximate nature of the calculation.

We make several sets of 16 quasi-independent $3^{\circ} \times 3^{\circ}$ maps with $1024^{2}$ pixels. The first set of maps has sources fixed at $z_{s} \equiv 1$, while the second set uses a 
source distribution of the form (Brainerd, Blandford \& Smail, 1996)

$$
\frac{d p}{d z_{s}} \propto z_{s}^{2} \exp \left[-\left(z_{s} / z_{0}\right)^{3 / 2}\right]
$$

for $z_{0}=2 / 3$ and 1 . For this distribution $\langle z\rangle=\Gamma\left(\frac{8}{3}\right) z_{0} \simeq 1.5 z_{0}$, but because there is more of the lensing weight at higher $z$ the lensing signal is smaller for this distribution than a $\delta$-function distribution with $z_{s}=1.5 z_{0}$.

The second set of maps comes from a full multi-plane ray tracing algorithm as described in Vale \& White (2003). We make maps of the shear components, $\gamma_{i}$, and the convergence, $\kappa$, at a range of source redshifts from $z \sim 0$ to 3 in steps of $50 \mathrm{~h}^{-1} \mathrm{Mpc}$. Again we make 16 maps per simulation, choosing different positions and orientations for the 'observer'. In each case a $2048^{2}$ grid of rays subtending a field of view of $3^{\circ}$ is traced through the simulation, with the required Fourier transforms being done on a $2048^{2}$ grid. All assignments to and from the Fourier grid are done with cloud-in-cell (CIC) assignment (Hockney \& Eastwood, 1980).

The two shear components and the convergence are output at each source plane. From this a map of the (measurable) reduced shear, $\gamma_{i} /(1-\kappa)$, for any source distribution can be computed. For a distribution $d p / d z_{s}$ the weight given to source plane $j$ is

$$
w_{j}=\left.\frac{d p}{d z_{s}}\right|_{j} H\left(z_{j}\right) \Delta \chi
$$

where $\Delta \chi=50 h^{-1} \mathrm{Mpc}$ is the spacing between outputs. The final shear at each point is then $\gamma_{i}=\sum_{j} w_{j} \gamma_{i}^{(j)}$ with $\gamma_{i}^{(j)}$ the $i$ th component of the shear measured on plane $j$. We show an example of the weighting factors for $z_{0}=1$ in Fig. 2, along with the (trivial) weight for a $\delta$-function source distribution at $z_{s} \equiv 1.5$. The integral over $\chi_{s}$ is extremely well approximated by a sum over the output planes. These maps can thus be combined to produce lensing maps for a wide range of source distributions, allowing source tomography to be used. It is straightforward to introduce mock 'galaxies', with an appropriate distribution of intrinsic ellipticities, at this stage and produce mock catalogues. On each $\chi_{s}$ slice, the angular positions of the galaxies can be made to trace the dark matter with some fidelity, giving rise to source clustering effects. Reasonable choices produce angular correlations and redshift distributions for the 'galaxies' which match current data well. However the parameter space of such catalogues is large, so we have chosen instead to make available simple co-added shear maps which can be post-processed with a wide variety of simulated galaxy properties to make shear maps. To save space we provide the maps downsampled from $2048^{2}$ to $1024^{2}$. 


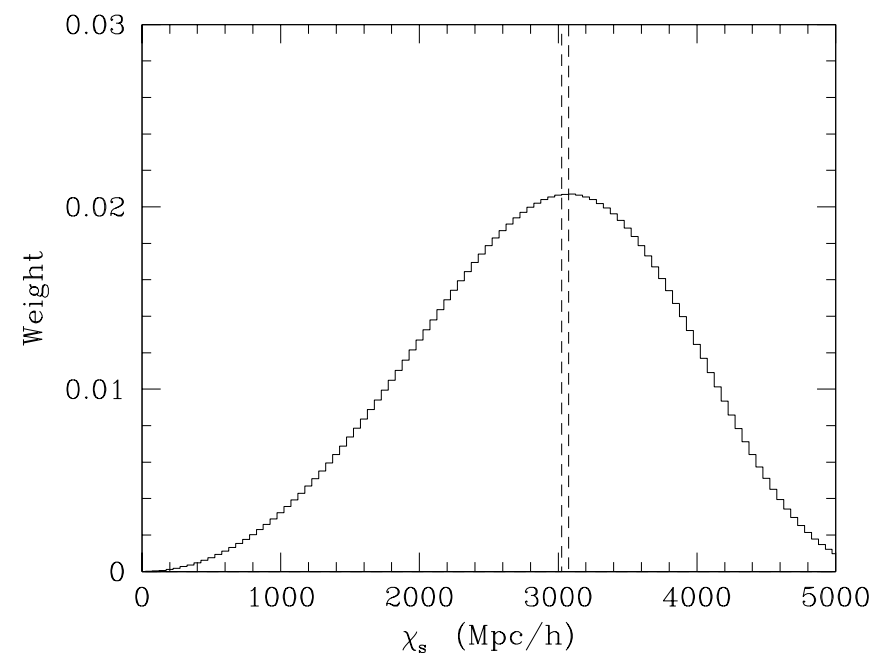

Fig. 2. An example of the weights to be applied to the source planes of model 1 in order to construct maps appropriate to Eq. (3) with $z_{0}=1$ (solid) and a trivial $z_{s} \equiv 1.5$ (dashed). The planes are spaced by $\Delta \chi=50 h^{-1} \mathrm{Mpc}$. The weight for $z_{s} \equiv 1.5$ is unity in a single bin. The area under the solid histogram is also unity, but $4 \%$ of this area lies beyond $z=3$ where our simulations stop.

\subsection{Numerics}

To understand the fidelity of the map making procedure we need to account for the finite volume, force and mass resolution of the N-body simulation and the effect of the finite sampling during ray tracing. The comoving distance to our furthest sources is $3 \mathrm{Gpc}$, at which distance our box subtends $3.8^{\circ}$. The volume of space probed by a lensing field is $\sim 10^{7} \mathrm{Mpc}^{3}$, comparable to the volume simulated in each run.

In Vale \& White (2003), we developed an analytic model to estimate the effects of finite force and mass resolution and the Fourier grid used in the ray tracing. This model was based on modifying the 3D dark matter power spectrum that appears in the Limber integral for $C_{\ell}$ in a way designed to fit the effects seen in a series of N-body simulations. This allows us to compare a given numerical configuration to a "perfect" model. Fig. 3 illustrates these effects individually for model 1 of Table 1 with sources at $z_{s} \equiv 1$. For simplicity we use the power spectrum fit of Smith et al. (2003) in computing these ratios. Note that the suppression of power by the finite force resolution and FT grid is partly countered by the artificial increase in power due to shot-noise. Based on Fig. 3 we expect our maps to be accurate to several percent, in the 2-point function, for $\ell \leq 3000$ and even higher in some situations where the cancellation is accurate.

While the 16 maps per model are not fully independent, they do sample dif- 


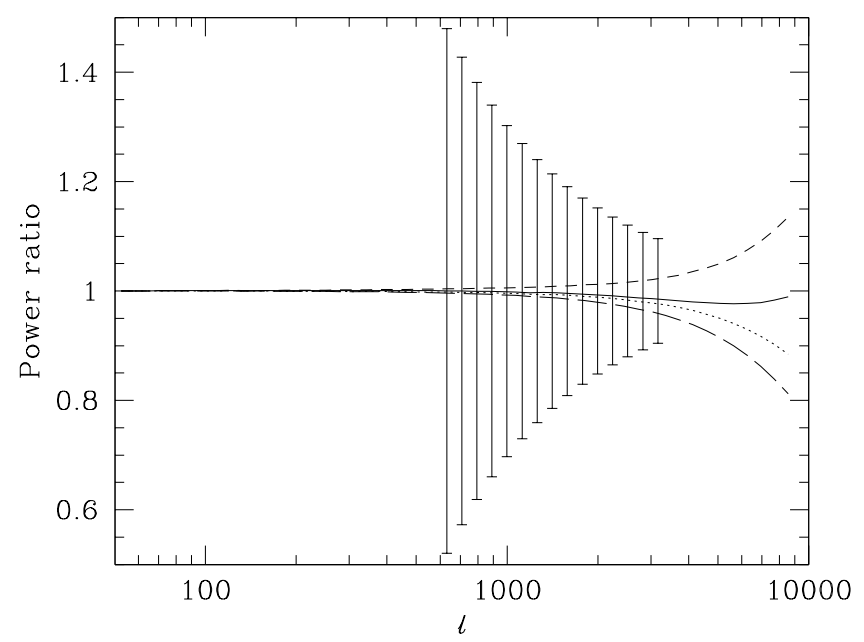

Fig. 3. Resolution effects in the power spectra of the maps, compared to the sample variance in a $3^{\circ} \times 3^{\circ}$ map. The $y$-axis shows the ratio of lensing power spectra to a fiducial model prediction. All calculations are done with the $3 \mathrm{D}$ mass power spectrum fit of Smith et al. (2003), the Limber approximation and the model of Vale \& White (2003). The dotted line shows the effect of the finite force resolution in the N-body simulation, the short-dashed line adds the finite mass resolution. The long-dashed line shows the effect of the FT grid in the lensing algorithm while the solid line combines all of these effects. The error bars indicate the sample variance expected in a $3^{\circ} \times 3^{\circ}$ field.

ferent regions of the box in different stages of evolution and with different projection effects. The fraction of the volume traced by the rays in the field of view, weighted by the contribution to the variance of the convergence from each box, is between 10-20\% (for $100<\ell<3000$ ). This forms a rough upper limit to the degree of correlation between the maps. Including the two independent boxes per model we have simulated several hundred square degrees of sky per cosmology, which is close to the amount of observational data currently existing. Extending this to more sky is a straightforward exercise that simply requires more computing resources.

\section{Results}

These simulations and lensing maps can be used for a variety of purposes. Here we simply show some preliminary analysis to orient the reader. The real uses of these maps will be in testing analysis algorithms and new ideas.

Before turning to the lensing maps let us consider the underlying mass distribution. Fig. 4 shows the 3D matter power spectrum from our 8 boxes at $z=1$ and $z=0$ compared to the semi-analytic model of Smith et al. (2003). The 


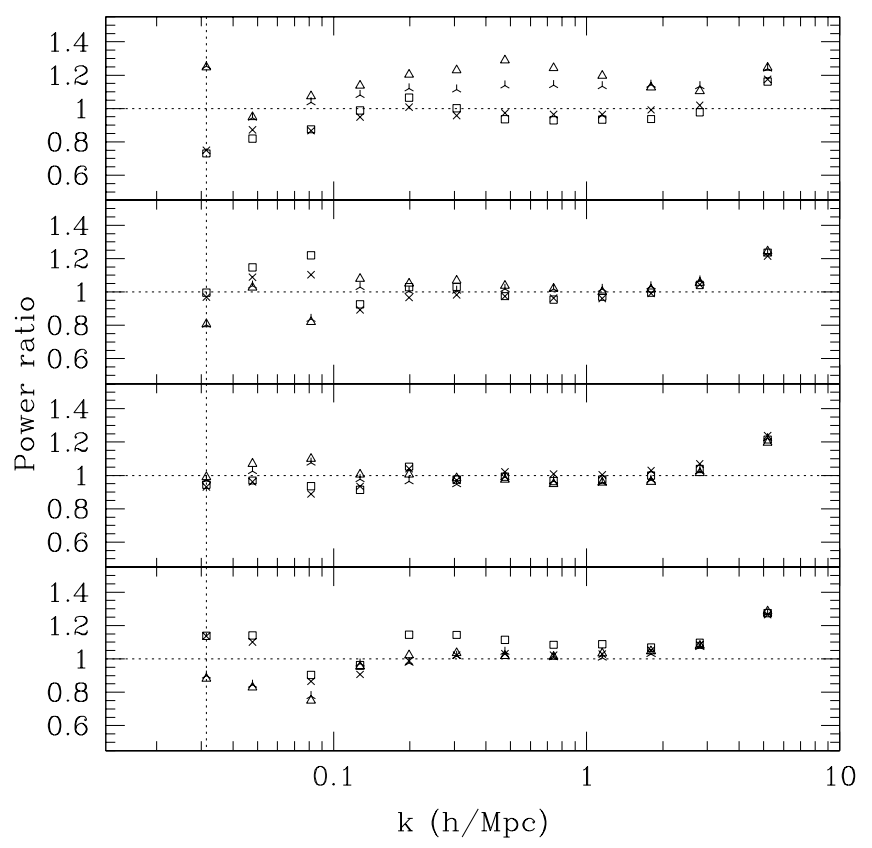

Fig. 4. A comparison of the 3D mass power spectrum with the fitting function of Smith et al. (2003) at selected redshifts. The $y$-axis shows the ratio of the numerical to the semi-analytic result, with the two independent runs per cosmology and two different redshifts shown as different symbol types in each panel. Open symbols are for $z=0$ and starred symbols are for $z \simeq 1$. The top panel is models 1 and 2 , then 3 and 4 and so on. The vertical dotted line marks the fundamental mode of the simulations.

agreement is good for both the $\Lambda$ CDM models and the models with equation of state $w=-0.8$, as expected. On the scales simulated the dark energy only enters through the Hubble parameter in the drag term, slowing the growth of large-scale fluctuations when the dark energy comes to dominate the expansion. Since the fit of Smith et al. (2003) has been tested for open and $\Lambda$ CDM models it is no surprise that it works equally well for $w=-0.8$. The disagreement at the highest $k$ might be due to numerical artifacts in the N-body simulation or in the computation of the power spectrum, however these points are significantly above both the shot-noise and force smoothing limits. We find similar behavior in a number of higher resolution simulations we have analyzed, and a similar level of disagreement can be found in some of the figures of Smith et al. (2003) for CDM models and may reflect inherent inaccuracy in the fitting function. A qualitatively similar level of agreement was also found between particle-mesh simulations and the semi-analytic model by Ishak et al. (2003), though they were unable to probe very high $k$-modes due to limited force resolution.

As a further check on the N-body code we investigated the scaling of the power spectrum of an $n=-1$ self-similar model with the same numerical parameters as the models described. This suggests that the power spectrum 


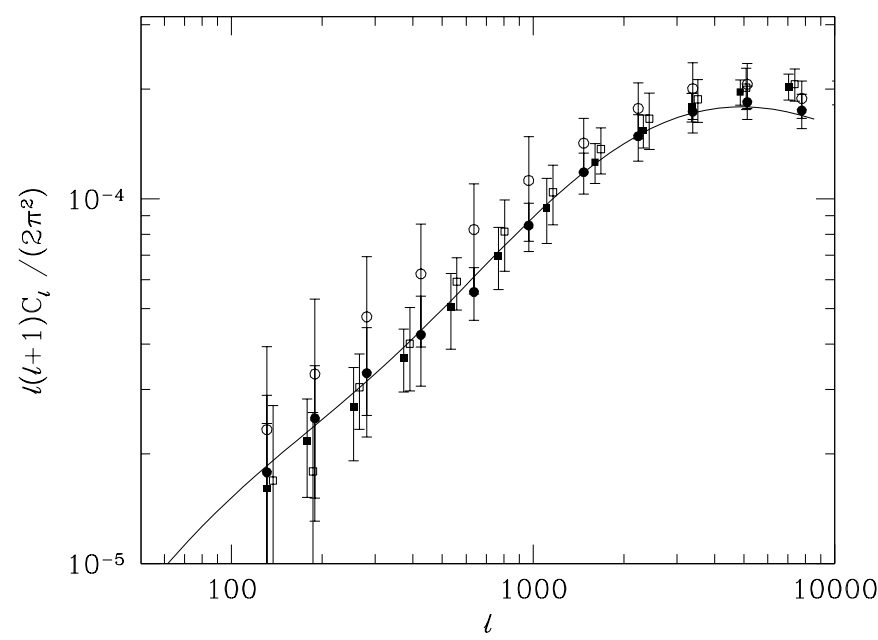

Fig. 5. The angular power spectra predicted for model 1 with $z_{s} \equiv 1$. The symbols with error bars are the mean and variance of the spectra from 16 maps, each $3^{\circ} \times 3^{\circ}$, from runs 1 and 2. The points have been offset slightly (horizontally) for clarity. The open and filled squares are the results from our "Born" series while the open and filled circles are for the full ray tracing.

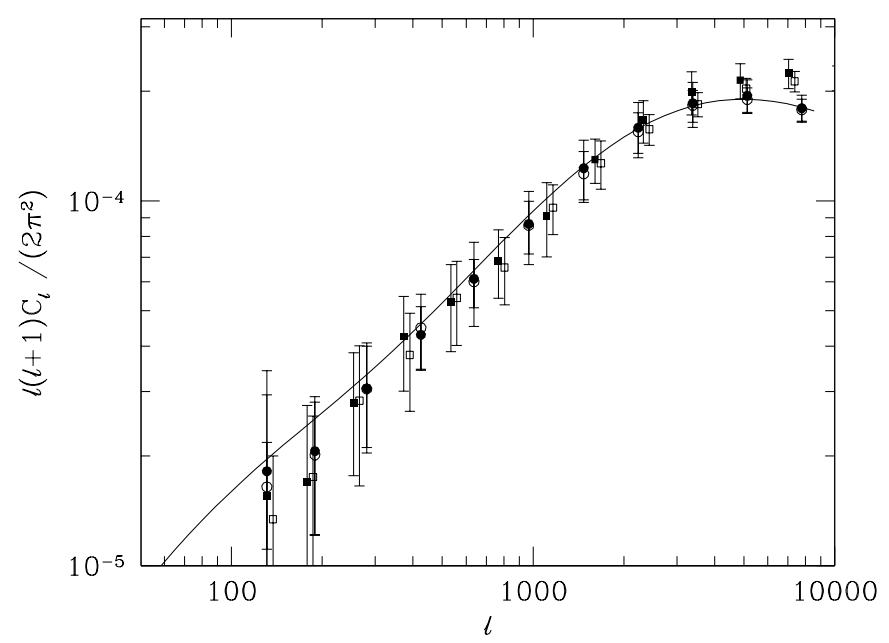

Fig. 6. The angular power spectra predicted for model 3 with $z_{s} \equiv 1$. The symbols with error bars are the mean and variance of the spectra from 16 maps, each $3^{\circ} \times 3^{\circ}$, from runs 3 and 4 . The points have been offset slightly (horizontally) for clarity. The open and filled squares are the results from our "Born" series while the open and filled circles are for the full ray tracing.

should be accurate to a few percent on the range of scales of interest.

The $\kappa$ angular power spectrum produced from 16 maps each for models $1 \& 2$ is shown in Fig. 5. For this comparison we show maps made assuming the Born approximation and with full ray-tracing, both for sources fixed at $z_{s} \equiv 1$. The agreement is generally good, with the variance at low- $\ell$ expected from sampling 


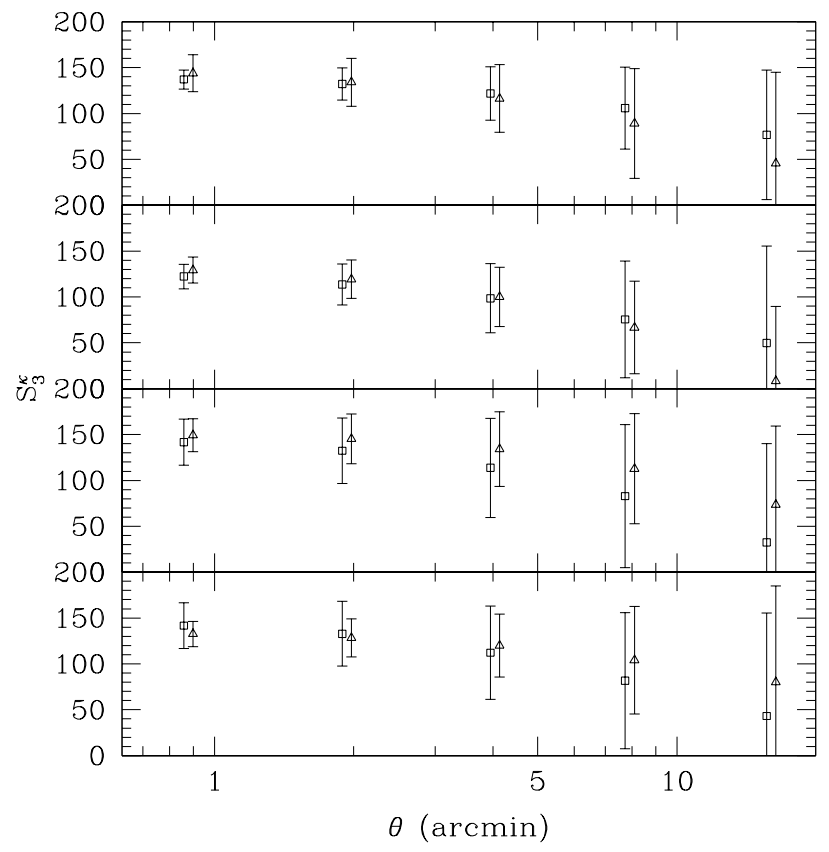

Fig. 7. The skewness of the convergence, $\kappa$, for models $1-8$ for sources at $z_{s} \equiv 1$. The symbols with error bars are the mean and variance of $S_{3}$ from 16 'Born' maps, each $3^{\circ} \times 3^{\circ}$. The results from the ray tracing are very similar and are omitted. The points have been offset slightly (horizontally) for clarity.

$163^{\circ} \times 3^{\circ}$ fields. The open circles, corresponding to model 2 , are slightly higher than the semi-analytic model at intermediate $\ell$, as expected from the excess power seen in the top panel of Fig. 4 (the triangles) at intermediate $k$. At the highest $\ell$ the 'Born' maps show a slight excess power which could be due to shot-noise in the simulations or to a breakdown in some of the approximations we have made. Alternatively the semi-analytic model and the ray tracing could be underestimating the power on these scales, as suggested by Figs. 3 and 4. In measurements these scales would be significantly affected by finite galaxy ellipticities and densities, so this disagreement is likely unimportant. Fig. 6 shows the same comparison for models 3 and 4 . The power spectra for the remaining four models are very similar and we do not show them explicitly here.

The maps are non-Gaussian, as expected, and we provide the skewness

$$
S_{3}^{\kappa} \equiv \frac{\left\langle\kappa^{3}\right\rangle}{\left\langle\kappa^{2}\right\rangle^{2}}
$$

for each of the models in Fig. 7. The $\kappa$ field is smoothed by a boxcar of side length $\theta$ before the moments are computed. The plot shows the average and the variance across the 16 maps as before. As noted previously (White \& $\mathrm{Hu}$, 2000; Vale \& White, 2003) the skewness suffers from a large sample variance. 


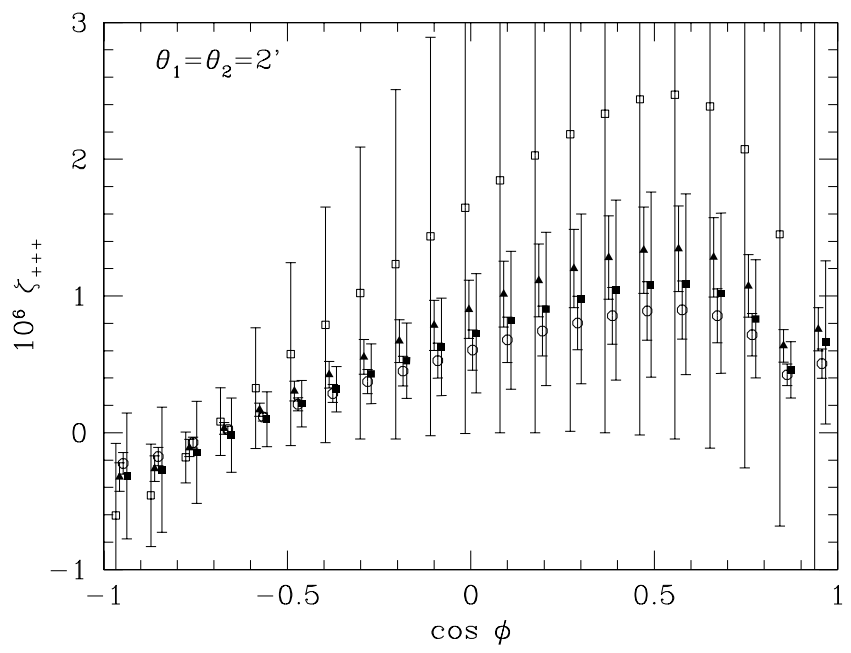

Fig. 8. The shear 3-point function, $\zeta_{+++}$, for isoceles triangles with $\theta_{1}=\theta_{2}=2^{\prime}$ as a function of the cosine of the included angle. Different symbol types represent the mean and standard deviation of 32 maps, each $3^{\circ} \times 3^{\circ}$, for the 4 cosmologies simulated: models $1 \& 2$ (open squares), $3 \& 4$ (filled triangles), $5 \& 6$ (open circles), $7 \& 8$ (filled squares). Points have been offset slightly horizontally for clarity. In each case we assume $z \equiv 1$.

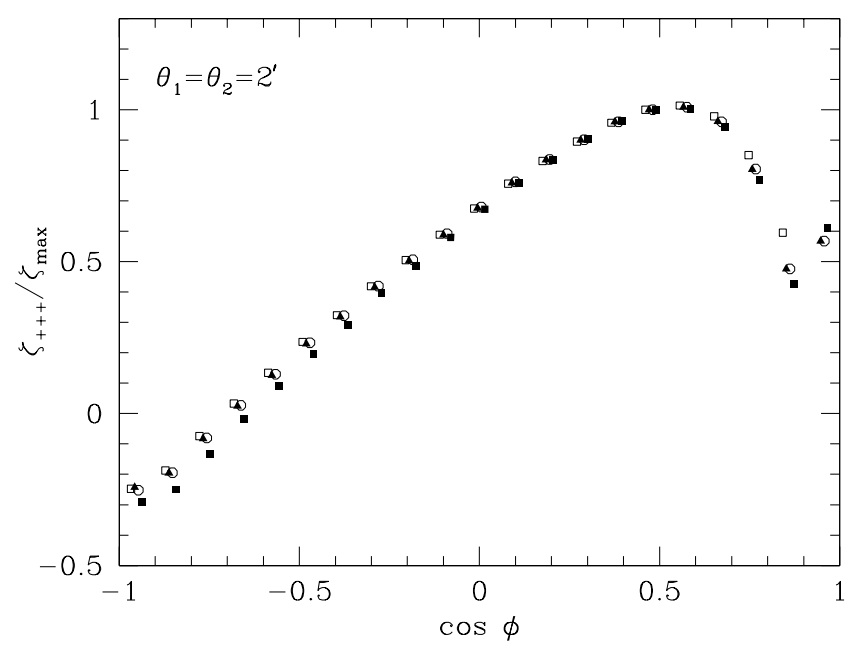

Fig. 9. The shear 3-point function, $\zeta_{+++}$, for isoceles triangles with $\theta_{1}=\theta_{2}=2^{\prime}$ as for Fig. 8, except scaled to unity at maxmimum. Note that the shape of the curves is very similar among all of the models. Symbol types are as in Fig. 8.

We also show the shear 3-point function for isoceles triangles of side length $\theta_{1}=\theta_{2}=2^{\prime}$ in Fig. 8. Here we plot $10^{6} \zeta_{+++}$as a function of the cosine of the included angle for the 32 fields, each $3^{\circ} \times 3^{\circ}$, simulated for each cosmology assuming the sources are at $z_{s} \equiv 1$. The points are the mean of the 32 fields, and the error bars represent the variance. The highest 3-point function is for models $1 \& 2$, which have the highest normalization $\left(\sigma_{8}\right)$ of all of the 
models. Models $3 \& 4$ have the next largest amplitude, and the second highest normalization. For models $5 \& 6$ the effect of a higher normalization isn't enough to overcome the less negative $w$ of models $7 \& 8$, which have a slightly higher amplitude. Notice the large scatter from field to field, but the familiar shape (Takada \& Jain, 2002). Apart from an amplitude change the shapes of the curves are extremely similar, as shown in Fig. 9. We find a similar behavior for other configurations we have checked, which has interesting implications for the signal encoded in the non-Gaussianity of the maps and how best to extract it. We also note that the peak of $\zeta_{+++}$appears to be shifted slightly from the equilateral configuration, due to the asphericity and substructure of the dark matter halos (Ho \& White, 2003; Dolney, Jain \& Takada, 2004).

\section{Conclusions}

Gravitational lensing has made rapid advances in recent years, and observations are now pushing the limits of existing theoretical models. If lensing is to fulfill its promise as a precision tool, theorists need to improve the predictions of cosmological models for the $n$-point correlation functions. An important step in this program is the creation of grids of N-body models of sufficient resolution and sampling to enable accurate simulation of weak lensing. This paper describes a first step in this direction.

Based on analytic arguments developed in Vale \& White (2003) we believe that simulations with $256^{3}$ particles in boxes of side $200 h^{-1} \mathrm{Mpc}$ are sufficient to produce lensing maps accurate to $\ell \simeq 2000-3000$ or scales of a few arcminutes. To achieve good convergence in the multi-plane ray-tracing algorithm that we use requires time dumps spaced equally in conformal time with a spacing close to $50 h^{-1} \mathrm{Mpc}$.

The total computational cost of this project was very modest. The major human cost is the time spent managing the disk space, archiving and retrieving the data to ensure that the total usage stays below quota. The simulations each took 2000-3000 time steps, requiring slightly under one day each on 32 processors of the IBM-SP Seaborg at NERSC, i.e. between 640 and 720 $\mathrm{CPU}$ hours. The phase space data required $\sim 40 \mathrm{~GB}$ per model while the ray tracing simulations required around $100 \mathrm{~GB}$ of intermediate storage and $100 \mathrm{CPU}$ hours per model. The disk usage is driven by the need for all the phase space data and the $80-90$ high resolution source planes for each of 16 maps. The final stacked and downsampled maps are $<1 \mathrm{~GB}$ per model and source distribution. The entire process was monitored and controlled by scripts, making it easy to extend the grid as more resources become available.

We have made the raw maps, along with some auxiliary data products, freely 
available to the community at http://mwhite.berkeley.edu/ in the hope that they will be useful in taking the next step.

M.W. thanks Tzu-Ching Chang, Dragan Huterer, Bhuvnesh Jain, Jason Rhodes and Masahiro Takada for helpful comments on an earlier draft. The simulations used here were performed on the IBM-SP at the National Energy Research Scientific Computing Center. This research was supported by the NSF and NASA.

\section{References}

Abazajian K., Dodelson S., 2003, PRL, 91, 041301

Bartelmann M., Schneider P., 2001, Phys. Rep., 340, 291

Benabed K., Bernardeau F., 2001, PRD, 64, 3501

Bernstei, G., Jain B., 2004, Astrophys. J., 600, 17 [astro-ph/0309332]

Brainerd T., Blandford R.D., Smail I., 1996, Astrophys. J., 466, 623

Dolney D., Jain B., Takada M., 2004, submitted to MNRAS [astro$\mathrm{ph} / 0401089]$

Eisenstein D., Hu W., 1999, Astrophys. J., 511, 5

Heavens A., 2003, Mon.Not.Roy.Astron.Soc., 343, 1327 [astro-ph/0304151]

Hinshaw G. et al., 2003, Astrophys. J. Supp., 148, 63

Ho S., White M., 2003, Astrophys. J., in press [astro-ph/0312253]

Hockney R.W., Eastwood J.W., "Computer simulation using particles", New York, McGraw Hill (1980).

Hoekstra H., Yee H.K.C., Gladders M.D., 2002, New Astronomy Reviews, 46, 767

Hu, W., 2002, PRD 65, 023003 ibid, 66, 083515

Huterer D., White M., 2002, Astrophys. J., 578, L95 [astro-ph/0206292]

Huterer D., 2002, PRD, 65, 3001

Ishak M., Hirata C.M., McDonald P., Seljak U., 2003, preprint [astro$\mathrm{ph} / 0308446]$

Jain B., Taylor A., 2003, PRL, 91, 141302

Kogut A. et al., 2003, Astrophys. J. Supp., 148, 161

Mellier Y., 1999, Ann. Rev. Astron. Astrophys., 37, 127

Refregier A. et al., 2003, Astrophys. J., in press [astro-ph/0304419]

Seljak U., Zaldarriaga M., 1996, Astrophys. J., 469, 437

Takada M., Jain B., 2002, MNRAS, 344, 857 [astro-ph/0209167]

Takada M., Jain B., 2004, MNRAS 348, 897 [astro-ph/0310125]

Takada, M., White M., 2004, Astrophys. J., 601, L1 [astro-ph/0311104]

Tegmark M. et al., 2003, preprint [astro-ph/0310723]

Seljak U., Sugiyama N., White M., Zaldarriaga M., 2003, PRD 68, 083507 [astro-ph/0306052]

Smith R.E. et al., 2003, MNRAS, 341, 1311 [astro-ph/0207664] 
Vale C., White M., 2003, Astrophys. J., 592, 699 [astro-ph/0303555]

Verde L. et al., 2003, Astrophys. J. Supp., 148, 195

van Waerbeke L., Mellier Y., 2003, preprint [astro-ph/0305089]

White M., Hu W., 2000, Astrophys. J., 537, 1 [astro-ph/9909165]

White M., 2002, Astrophys. J. Supp., 143, 241 [astro-ph/0207185] 